\title{
ON THE COMMUTATIVITY AND ANTICOMMUTATIVITY OF RINGS II'
}

\author{
CHEN-TE YEN
}

\begin{abstract}
It is shown that if $R$ is any associative ring such that for each $x, y \in R$, there exist an even natural number $m(x, y)$ and an odd natural number $n(x, y)$, depending on $x$ and $y$, with either $[x, y]^{m(x, y)}=[x, y]^{n(x, y)}$ or $(x \circ y)^{m(x, y)}=$ $(x \circ y)^{n(x, y)}$, then either $[x, y]$ or $(x \circ y)$ is nilpotent for all $x, y$ in $\mathbb{R}$. Moreover, $\mathbb{R}$ is commutative if $\mathbb{R}$ has no nonzero nil right ideals.
\end{abstract}

\section{Introduction}

Throughout the paper, $R$ will denote an associative ring with Jacobson radical $J(R)$. For $x, y \in R$, set $[x, y]=x y-y x$ and $(x \circ y)=x y+y x$. $R$ is called commutative (resp. anticommutative) if $[x, y]=0$ (resp. $(x \circ y)=0)$ for all $x, y$ in $R$. $R$ is called semiprime if for $a \in R, a R a=0$ implies that $a=0$. A right ideal $A$ of $R$ is called nil if for every $a \in A, a^{n}=0$ for some positive integer $n=n(a)$. An element $x$ in $R$ is called periodic (resp. strongly periodic) if there exists an integer $n>1$ (resp. an even positive integer $m$ ), with $x^{n}=x$ (resp. $x^{m}=x$ ). An element $x$ of $R$ is called (*)-periodic if there exist an even natural number $m$ and an odd natural number $n$ such that $x^{m}=x^{n}$. Note that if char $R=2$, then $R$ is commutative if and only if $R$ is anticommutative.

In 1957, Herstein [1] prove

Theorem $\mathbb{A}$. If for each $x, y \in R$, there exists a natural number $n(x, y)>1$, depending on $x$ and $y$, with $[x, y]^{n(x, y)}=[x, y]$, then $R$ is commutative.

In 1987, Machale [3] proved

Theorem $\mathbb{B}$. If for each $x, y \in R$, there exists an even natural number $m(x, y)$, depending on $x$ and $y$, with $(x \circ y)^{m(x, y)}=(x \circ y)$, then $R$ is anticommutative.

Later in 1987, Yen [4] combined Theorem A and Theorem B to obtain

Received October 2, 1990.

1980 Mathematics Subject Classification (1985 Revision).

Primary 16A70.

Keywords and phrases: Jacobson radical, nil ideal, division, primitive, semisimple, semiprime ring. 
Theorem. C. Suppose that for each $x, y \in R$, there exists either a natural number $n(x, y)>1$ such that $[x, y]^{n(x, y)}=[x, y]$ or an even natural number $m(x, y)$ such that $(x \circ y)^{m(x, y)}=(x \circ y)$. Then for each $x, y \in R$,

(1) $[x, y]=0$, if $[x, y]$ is periodic;

(2) $x \circ y=0$ if both $(x \circ y)$ and $((-y) \circ x)$ are strongly periodic.

Furthermore, $R$ is either commutative or anticommutative; in particular, $R$ is commutative if $R$ is semiprime.

The purpose of this note is to generalize Theorem B and partially extend Theorem $\mathrm{A}$, and combine these generalizations.

\section{Results}

Lemma 1. If for each $x, y \in R$, there exist an even natural number $m(x, y)$ and an odd natural number $n(x, y)$, with $[x, y]^{m(x, y)}=[x, y]^{n(x, y)}$, then $[x, y]$ is nilpotent for all $x, y \in R$. Moreover, $R$ is commutative if it has no nonzero nil right ideals.

Proof. If $R$ is a division ring, then by Herstein's theorem [1, or 2, Theorem 3.1.3], $R$ is commutative.

If $R$ is a primitive ring, then the first possibility is that $R \cong D$, where $D$ is a division ring, and so $R$ is a field. Otherwise, for some $k>1, D_{k^{\prime}}$ the complete matrix ring over a division ring $D$, would be a homomorphic image of a subring of $R$. Thus $D_{k}$ would inherit the property $[x, y]^{m(x, y)}=[x ; y]^{n(x, y)}$, where $m(x, y)$ and $n(x, y)$ are as above. Let $e_{i j}$ 's be the matrix units of $D_{k}$. In $D_{k^{\prime}}$ let $x=e_{11}+e_{12}$ and $y=-e_{21}$. Then $[x, y]=e_{21}+e_{22}-e_{11}$. Hence

$$
\begin{aligned}
& {[x, y]^{m}=e_{11}+e_{22} \quad \text { if } m \text { is an even positive integer, and }} \\
& {[x, y]^{n}=e_{21}+e_{22}-e_{11} \quad \text { if } n \text { is an odd positive integer. }}
\end{aligned}
$$

This yields a contradiction. Therefore, $R$ is a field.

If $R$ is a semisimple ring, then $R$ is isomorphic to a subdirect sum of primitive rings $R_{i}$, each of which, as a homomorphic image of $R$, satisfies the hypotheses placed on $R$. Thus each $R_{i}$ is a field by the result above, and we conclude that $R$ is commutative.

Now, let $R$ be any ring satisfying the hypotheses. Since $R / J(R)$ is a semisimple ring, by the previous result $R / J(R)$ is commutative. Hence, $[x, y] \in J(R)$ for all $x, y$ in $R$. Now $J(R)$ has the property that if $a b=a$, with $b \in J(R)$, then $a=0$. Therefore, $[x, y]^{m(x, y)}=[x, y]^{n(x, y)}$ implies that $[x, y]^{n(x, y)}=0$ for all $x, y$ in $R$.

Finally, let $R$ be a ring having no nonzero nil right ideals. Since $R$ has no nonzero nil right ideals, $R$ is semiprime. To prove that $R$ is commutative, it suffices to show that if $a \in R$ and $a^{2}=0$ then $a=0$. For every $x \in R$, we have $0=[a, x]^{n(a, x)}=[a, x]^{n(a, x)} a$. Thus, $(a x)^{n(a, x)+1}=0$. So, $a R$ is a nil right ideal. Ience, $a R=0$. By semiprimeness of $R, a R=0$ implics that $a=0$.

Lomma 2. If for each $x, y \in R$, there exist an cven natural number $m(x, y)$ and an odd natural number $n(x, y)$, with $(x \circ y)^{m(x, y)}=(x \circ y)^{n(x, y)}$, thcn $(x \circ y)$ is nilpotent for 
all $x, y \in R$. Moreover, $R$ is anticommutative and char $R=2$ if it has. no nonzero nil right ideals.

Proof. If $R$ is a division ring, then $R$ is anticommutative by Machale's theorem [3]. If $R$ is a primitive ring, then the first possibility is that $R \cong D$, where $D$ is a division ring, and so $R$ is anticommutative. Otherwise, for some $k>1, D_{k}$ the complete matrix ring over a division ring $D$, would be a homomorphic image of a subring of $R$. Thus $D_{k}$ would inherit the property $(x \circ y)^{m(x, y)}=(x \circ y)^{n(x, y)}$, where $m(x, y)$ and $n(x, y)$ are as above. Let $e_{i j}$ 's be the matrix units of $D_{k}$. In $D_{k}$ let $x=e_{11}+e_{12}$ and $y=-e_{21}$. Then $(x \circ y)=-e_{11}-e_{21}-e_{22}$. Hence $(x \circ y)^{m}=e_{11}+m e_{21}+e_{22}$ if $m$ is an even positive integer, and $(x \circ y)^{n}=-e_{11}-n e_{21}-e_{22}$ if $n$ is an odd positive integer. Thus, the hypotheses imply that char $D=2$. This yields a contradiction. So, $R$ is anticommutative.

If $R$ is a semisimple ring, then. $R$ is isomorphic to a subdirect sum of primitive rings $R_{i}$, each of which, as a homomorphic image of $R$, satisfies the hypotheses placed on $R$. Thus each $R_{i}$ is anticommutative by the result above, and we conclude that $R$ is anticommutative.

Now, let $R$ be any ring satisfying the hypotheses. Since $R / J(R)$ is a semisimple ring, by the previous result $R / J(R)$ is anticommutative. Hence, $(x \circ y) \in J(R)$ for all $x, y$ in $R$. Therefore, $(x \circ y)^{m(x, y)}=(x \circ y)^{n(x, y)}$ implies that $(x \circ y)^{n(x, y)}=0$ for all $x, y$
in $R$.

Finally, let $R$ be a ring having no nonzero nil right ideals. Then as the proof of Lemma $1, R$ is anticommutative. Hence, $2 x^{2}=(x \circ x)=0$ for all $x$ in $R$. Thus, $2 R$ is a nil right ideal and so $2 R=0$.

By the proofs of Lemma 1 and Lemma 2, we have the following

Lemma 3. Suppose that for each $x, y \in R$, there exist an even natural number $m(x, y)$ and an odd natural number $n(x, y)$ such that either

$$
[x, y]^{m(x, y)}=[x, y]^{n(x, y)}
$$

or

$$
(x \circ y)^{m(x, y)}=(x \circ y)^{n(x, y)} .
$$

If $R$ is a semisimple ring, then $R$ is commutative.

Proof. If $R$ is a division ring, then by Lemma 3 of [4], $R$ is commutative.

If $R$ is a primitive ring, then as the proofs of Lemma 1 and Lemma 2, we can show that $R$ is commutative.

If $R$ is a semisimple ring, then as the proof of Lemma 1 , we can prove that $R$ is commutative.

Theorem. Suppose that for each $x, y \in R$, there exist an even.natural number $m(x, y)$ and an odd natural number $n(x, y)$ such that either $[x, y]^{m(x, y)}=[x, y]^{n(x, y)}$ or
$(x \circ y)^{m(x, y)}=(x \circ y)^{n(x, y)}$. Then for each $x, y \in R$,

(1) $[x, y]$ is nilpotent, if $[x, y]$ is (*)-periodic; 
(2) $(x \circ y)$ is nilpotent if both $(x \circ y)$ and $((-y) \circ x)$ are (*)-periodic.

Furthermore, $R$ is commutative if $R$ has no nonzero nil right ideals.

Proof. If $R$ is a semisimple ring, then by Lemma $3, R$ is isomorphic to a subdirect sum of fields.

Now, let $R$ be any ring satisfying the hypotheses. Let $x, y \in R$. Since $[x, y]=[-y, x]$, it suffices to consider the following two cases:

Case 1. $[x, y]$ is (*)-periodic. Since $R / J(R)$ is a semisimple ring, by Lemma 3, $R / J(R)$ is commutative. Thus, $[x, y] \in J(R)$. Hence, $[x, y]^{m(x, y)}=[x, y]^{n(x, y)}$ implies that $[x, y]^{n(x, y)}=0$.

Case 2. $(x \circ y)$ and $((-y) \circ x)$ are $(*)$-periodic. Consider the semisimple ring $\bar{R}=R / J(R)$. By the result above, $\bar{R}$ is isomorphic to a subdirect sum of fields $R_{i}$, $i$ in some index set $I \neq \phi$. Let $\mathbb{\Pi}_{j \in I} R_{j}$ be the complete direct sum, and $\phi: \bar{R} \rightarrow \mathbb{\Pi}_{j \in I} R_{j}$ be a monomorphism. Let $\Pi_{i}$ be the projection of $\Pi_{j \in I} R_{j}$ onto $R_{i}$, then by definition, $\bar{R} \phi \Pi_{i}=R_{i}$ for each $i \in I$. Thus, $(x \circ y)$ is (*)-periodic implying that $\left(\bar{x} \phi \Pi_{i} \circ \bar{y} \phi \Pi_{i}\right)$ is (*)-periodic for each $i \in I$. Since $R_{i}$ is a field, it follows that $\left(\bar{x} \phi \Pi_{i} \circ \bar{y} \phi \Pi_{i}\right)$ is strongly periodic for each $i \in I$. Similarly, $\left(\left(-\bar{y} \phi \Pi_{i}\right) \circ \bar{x} \phi \Pi_{i}\right)$ is strongly periodic for each $i \in I$. By Theorem $C,(\bar{x} \circ \bar{y}) \phi \Pi_{i}=\left(\bar{x} \phi \Pi_{i} \circ \bar{y} \phi \Pi_{i}\right)=0$ for each $i \in I$. So, $(\bar{x} \circ \bar{y}) \phi=0$. Since $\phi$ is one to one, $(\bar{x} \circ \bar{y})=0$, i.e., $(x \circ y) \in J(R)$. Hence, $(x \circ y)^{m(x, y)}=(x \circ y)^{n(x, y)}$ implies that $(x \circ y)^{n(x, y)}=0$.

Finally, let $R$ have no nonzero nil right ideals. Then as the proofs of Lemma 1 and Lemma 2, we have that either $[x, y]=0$ or $(x \circ y)=0$ for all $x, y$ in $R$. Thus, $R$ is commutative by the result of [4].

Finally, we note that Lemma 1 does not hold if both of $m(x, y)$ and $n(x, y)$ are even or odd. To see this, let $F$ be a finite field. Observing that $[x, y]^{2}$ is a scalar matrix for all $x, y$ in $F_{2}$, it is easy to see that the hypotheses as in Lemma 1 hold. However, $[x, y]$ is not necessarily nilpotent for all $x, y$ in $F_{2}$. We also note that Lemma 2 does not hold if both of $m(x, y)$ and $n(x, y)$ are even or odd.

\section{References}

[1] I. N. Herstein, "A condition for the commutativity of rings", Canad. J. Math., 9, 583-586, 1957.

[2] I. N. Herstein, "Noncommutative rings", Curus Mathematical Monographs, No. 15, Mathematical Association of America, Washingt.on, DC, 1968. Amer. Math. Monthly, 94, 162-165, 1987.

[4] C. T. Yen, "On the commutativity and anticommutativity of rings", Chinese J. Math., 15, 231-235,
1987 . 